\title{
Direct numerical simulation of noise suppression by water injection in high-speed flows
}

\author{
Jesse Capecelatro*, \\ University of Michigan, Ann Arbor, Michigan, 48109-2125, USA \\ and \\ David A. Buchta \\ University of Illinois at Urbana-Champaign, Urbana, Illinois, 61801, USA
}

\begin{abstract}
The injection of micro-water droplets in high-speed shear-flow turbulence reduces the radiated near-field pressure fluctuations, and the mechanisms responsible for this observed acoustic suppression remain elusive. Direct numerical simulation of droplet-laden highspeed free-shear flows are used to evaluate the effect of particle-turbulence interactions on the near-field acoustics. A Mach 1.5 temporally-developing mixing layer is seeded with $\mathcal{O}\left(10^{7}\right)$ water droplets concentrated in the high-shear region for a range of mass loadings. The reduction in turbulence fluctuations increases with the mass loading, with a concomitant decrease in the very near-field radiated pressure fluctuations. Up to a $2.5 \mathrm{~dB}$ reduction is observed, consistent with experimental observations under similar operating conditions.
\end{abstract}

\section{Introduction}

Aeroacoustic noise suppression in high-speed flows is a key design challenge for supersonic aircraft, including military fighter planes and potentially supersonic business jets, and also flow conditions that are applicable to space launches. ${ }^{1-3}$ The loud sound from jet noise can pose health risks to personnel working in close proximity. In addition, stringent regulations on environmental noise pollution require quieter jets. For even faster and larger-scale jets engines, such as those on space vehicles, unaccounted for aeroacoustic loading on the fuselage and nearby equipment can be catastrophic for extreme-risk space programs. Experiments of water-droplet injection in high-speed shear-flow turbulence show a reduction in their near-field pressure fluctuations. ${ }^{3-7}$ Water sound suppression systems are also being used for launch vehicle liftoff. However, the nonlinear and multiscale nature of turbulent particle-laden flows leads to significant challenges in understanding its effects on radiated sound.

The primary sources of noise associated with these high-speed flows are from the turbulence in the highshear region. ${ }^{8,9}$ The noise-reduction technique via micro-jet injection targets this region by introducing a spray of small $(4-140 \mu \mathrm{m})$ inertial water droplets that interact with the shear-flow turbulence. Experimental observations indicate that particle-turbulence interactions reduce the normal velocity fluctuations which is seen to correlate to a reduction in the near-field noise. ${ }^{4}$ This reduction also appears linked to the reduction of pressure skewness in the near field, ${ }^{4}$ an indicator of jet crackle, ${ }^{10}$ and confirmed by single phase (unladen) simulations of this kind. ${ }^{11}$ To date, experimental studies have demonstrated moderate acoustic suppression using water injection, but high-resolution diagnostics required for these high-speed multiphase flows do not yet exist.

While turbulence modulation by droplets is known to affect radiated acoustics, its sound reducing mechanisms are not well understood enough to improve design of current systems. Many open questions remain that cannot be answered by experiments alone, including 1) what direct mechanisms associated with particleturbulence coupling are responsible for acoustic suppression? 2) to what extent can the noise levels be reduced, and 3) how does noise reduction depend on the particle loading and injection velocity? As stated

*Assistant professor. Mechanical Engineering. AIAA member.

${ }^{\dagger}$ Postdoctoral researcher. The Center for Exascale Simulation of Plasma-coupled Combustion. AIAA student member 
in an experimental study by Krothapalli et al. ${ }^{4}$ "The results discussed in this paper clearly demonstrate the viability of water injection for noise suppression; however, several issues must be addressed. Since [direct numerical simulation] studies have advanced to the extent of predicting radiated noise levels from both high subsonic and supersonic jets, it would be of great interest to see if such computations can show significant noise suppression due to the use of water injection?"

In the past two decades, direct numerical simulation (DNS) is a viable tool for studying particle-turbulence interactions. DNS of inertial particles in isotropic turbulence ${ }^{12-16}$ and shearless mixing layers ${ }^{17}$ show the tendency for particles to preferentially concentrate in regions of high strain rate and low vorticity. At sufficient mass loading, DNS studies have demonstrated that momentum coupling between the phases can alter the underlying turbulent flow. ${ }^{18-22}$ While preferential concentration of inertial particles have been observed to directly affect fluid-phase velocity fluctuations, a detailed study on how this impacts near-field acoustics in compressible flows remains elusive.

To isolate the effects of particle-turbulence-acoustic coupling in high-speed flows, we perform DNS of Mach number $M a=1.5$ temporally developing mixing layers with particle mass loading varying from $M L=0$ to 2 . We consider moderate Stokes numbers and particle Reynolds numbers with up to 50 million particles at the highest mass loading. In the following section the flow configuration and governing equations are presented. Section III summarizes the velocity and pressure statistics, and the level of acoustic noise suppression is quantified and discussed.

\section{Simulation details}

\section{A. Governing equations}

The flow of rigid spherical particles suspended in a compressible viscous fluid is solved in an EulerianLagrangian framework, where the three-dimensional Navier-Stokes equations are solved for the non-dimensional fluid density $\rho$, momentum $\rho \boldsymbol{u}$, and the total energy $\rho E$. The fluid-phase conservation equations are given by

$$
\begin{gathered}
\frac{\partial \rho}{\partial t}+\nabla \cdot(\rho \boldsymbol{u})=0 \\
\frac{\partial \rho \boldsymbol{u}}{\partial t}+\nabla \cdot(\rho \boldsymbol{u} \otimes \boldsymbol{u}+p \mathbb{I}-\boldsymbol{\tau})=\boldsymbol{F}^{p}
\end{gathered}
$$

and

$$
\frac{\partial \rho E}{\partial t}+\nabla \cdot(\{\rho E+p\} \boldsymbol{u}+\boldsymbol{q}-\boldsymbol{u} \cdot \boldsymbol{\tau})=\boldsymbol{u} \cdot \boldsymbol{F}^{p},
$$

where $\mathbb{I}$ is the identity matrix. In this work, all variables are non-dimensionalized with the ambient density $\rho_{\infty}^{\star}$, speed of sound $c_{\infty}^{\star}$, a characteristic length scale $L^{\star}$, and heat capacity at constant pressure $C_{p}^{\star}$. All dimensional quantities are denoted by a superscript $\star$, and the subscript $\infty$ indicates a reference quantity (taken to be air at ambient conditions). The source term $\boldsymbol{F}^{p}$ appearing in Eqs.(2) and (3) contain contributions from momentum coupling with the particle phase, which will be made explicit later. The non-dimensional viscous stress tensor is defined as

$$
\boldsymbol{\tau}=\frac{\mu}{\operatorname{Re}_{c}}\left(\nabla \boldsymbol{u}+\nabla \boldsymbol{u}^{\top}\right)+\frac{\lambda}{\operatorname{Re}_{c}} \nabla \cdot \boldsymbol{u}
$$

and the heat flux $\boldsymbol{q}$ is given by

$$
\boldsymbol{q}=-\frac{\mu}{\operatorname{Re}_{c} \operatorname{Pr}} \nabla T,
$$

where $\operatorname{Pr} \equiv C_{p}^{\star} \mu^{\star} / k^{\star}$ is the Prandtl number, with $\mu^{\star}$ and $k^{\star}$ the dynamic viscosity and thermal conductivity, respectively. The Reynolds number used in the formulation is defined as $\operatorname{Re}_{c}=\operatorname{Re} / \mathrm{Ma}$, where $\operatorname{Re}=$ $\rho_{\infty}^{\star} \Delta U^{\star} L^{\star} / \mu_{\infty}^{\star}$ is the flow Reynolds number with $\Delta U^{\star}$ the velocity difference of the shear layer configuration described later, and $\mathrm{Ma}=\Delta U^{\star} / c_{\infty}^{\star}$ is the Mach number. The non-dimensional viscosity is modeled as a power law $\mu=[(\gamma-1) T]^{n}$, with $n=0.666$ as a model for air and $\gamma$ is the ratio of specific heats. The second coefficient of viscosity is given by $\lambda=\mu_{B}-\frac{2}{3} \mu$, where the bulk viscosity $\mu_{B}=0.6 \mu$ is chosen as a model for bulk viscosity of air. Finally, the thermodynamic pressure $p$ and temperature $T$ are obtained via the equation of state for an ideal gas, given in non-dimensional form as

$$
p=(\gamma-1)\left(\rho E-\frac{1}{2} \rho u_{i} u_{i}\right) \quad \text { and } \quad T=\frac{\gamma p}{(\gamma-1) \rho} .
$$


The displacement of an individual particle $i$ is calculated using Newton's second law of motion by

$$
\frac{d \boldsymbol{x}_{p}^{(i)}}{d t}=\boldsymbol{u}_{p}^{(i)} \text { and } \quad \frac{d \boldsymbol{u}_{p}^{(i)}}{d t}=\boldsymbol{F}_{d}^{(i)} .
$$

where $\boldsymbol{x}_{p}^{(i)}$ and $\boldsymbol{u}_{p}^{(i)}$ are the instantaneous position and velocity of the $i$-th particle, respectively. The particle equations are non-dimensionalized using the same reference quantities used in Eqs. (1)-(3). The classic Schiller and Naumann ${ }^{23}$ drag term is used on the right-hand side of Eq. (7) to account for finite Reynolds number effects, expressed as

$$
\boldsymbol{F}_{d}^{(i)}=\frac{1+0.15 \mathrm{Re}_{p}^{0.687}}{\tau_{p}}\left(\boldsymbol{u}\left[\boldsymbol{x}_{p}^{(i)}\right]-\boldsymbol{u}_{p}^{(i)}\right)
$$

where $\tau_{p}=\rho_{p} d_{p}^{2} \operatorname{Re}_{c} /(18 \mu)$ is the particle response time, with $\rho_{p}$ and $d_{p}$ the particle density and diameter, respectively, and $\operatorname{Re}_{p}=\rho d_{p} \operatorname{Re}_{c}\left\|\boldsymbol{u}-\boldsymbol{u}_{p}\right\| / \mu$ is the particle Reynolds number. Fluid quantities appearing in Eq. (8) (i.e., velocity, density, and viscosity) are interpolated to the location of each particle via a trilinear interpolation scheme. It is worth nothing that while the accuracy of a particle's displacement in time has been shown to be superior for higher order interpolation schemes in one-way coupled flows, ${ }^{24}$ a recent study ${ }^{25}$ showed that trilinear interpolation provides a better estimate of the undisturbed fluid velocity when the particle has a non-negligible effect on the local flow, leading to a more accurate prediction of the settling speed.

The interphase momentum transfer term appearing in Eqs. (2) and (3) is computed by linearly projecting the individual particle sources, $\boldsymbol{F}_{d}^{(i)}$, onto the eight neighboring computational nodes that make up the grid cell surrounding a given particle, i.e.,

$$
\boldsymbol{F}^{p}(\boldsymbol{x}, t)=-\frac{1}{V_{c}} \sum_{i=1}^{N_{p}} \mathcal{W}(\boldsymbol{x})^{(i)} m_{p} \boldsymbol{F}_{d}^{(i)},
$$

where $V_{c}$ is the volume of the computational cell, $m_{p}=\rho_{p} \pi d_{p}^{3} / 6$ is the particle mass and $N_{p}$ is the total number of particles. The contribution of each particle $i$ is weighted linearly to the node via $\mathcal{W}^{(i)}$ using an identical stencil as the interpolation procedure. As pointed out by Sundaram and Collins, ${ }^{26}$ the projection scheme should match the order of the velocity interpolation in order to ensure conservation of kinetic energy between phases, and thus the current scheme conserves mass, momentum, and energy in both phases.

\section{B. Discretization}

In the present study, spatial derivatives are approximated by narrow-stencil finite-difference operators that satisfy the summation-by-parts (SBP) property. ${ }^{27}$ An explicit, sixth-order, centered finite difference is used in the domain interior, and third-order, one-sided finite differences are applied at the boundary. The SBP scheme is combined with the simultaneous-approximation-term (SAT) ${ }^{28}$ approach at the domain boundaries in order to ensure an energy estimate so that the scheme is expected to be stable for long temporal integration. To evaluate the second and mixed derivatives, first-order derivative operators are applied consecutively, which necessitates the use of artificial dissipation, since the wide-stencil approximation does not damp the highest wavenumber supported by the grid. To dampen the high-wave number components which can potentially destabilize the numerical solution, high-order accurate SBP dissipation operators ${ }^{29,30}$ are used to provide artificial viscosity based on a sixth-order derivative with a diffusion coefficient that depends on the local grid resolution. Both the fluid and particle phases are advanced in time with a constant time step $\left(\Delta t=0.03 l^{\star} / c_{\infty}^{\star}\right)$ by a standard fourth-order explicit Runge-Kutta scheme. The Courant-Friedrichs-Lewy $(C F L)$ number was monitored throughout the simulation and remained below $C F L=0.5$.

\section{Flow configuration}

The simulations are designed to reproduce the key phenomenology of the full-scale droplet-injected jet applications to understand the details of particle-turbulence-acoustic coupling. To this end, we focus on the near-nozzle region of the shear layer. Since the equations are frame-invariant, we switch from an approximately planar spatially-developing shear layer to that of a temporally-developing frame of reference, as shown in Fig. 1. This enables us to explicitly resolve a broader range of turbulence scales than could be 
represented in a full jet simulation. The present configuration provides a Reynolds number realistic representation of a section of a high-Reynolds-number jet, and it therefore allows us to probe the sound generation mechanisms of high-Reynolds-number turbulence. Lab-scale measurements of the near-nozzle shear layers show that the momentum thickness is $\delta_{m}^{o} \sim 10^{-3} D_{j}$, with $D_{j}$ the jet diameter and $\delta_{m}^{o}$ the initial momentum thickness, suggesting that a low- $\operatorname{Re}_{\delta_{m}^{o}}$ DNS with $\operatorname{Re}_{\delta_{m}^{o}} \sim 10^{2}-10^{3}$ can represent a section of a moderately high $\operatorname{Re}_{D_{j}} \sim 10^{5}-10^{6}$ flow by

$$
\operatorname{Re}_{D_{j}} \equiv \frac{\rho_{\infty}^{\star} U_{j} D_{j}}{\mu^{\star}}=\operatorname{Re}_{\delta_{m}^{o}} \times \frac{D_{j}}{\delta_{m}^{o}} .
$$

We note that the acoustic far-field from the current mixing layers will not include any geometric propagation associated with a round jet, though this will not be a large effect for studying the very near field and their source mechanisms. Full details of the simulation configuration for the corresponding single-phase flow can be found elsewhere ${ }^{31}$ and details on the initialization of the turbulence can be found in Appendix A.

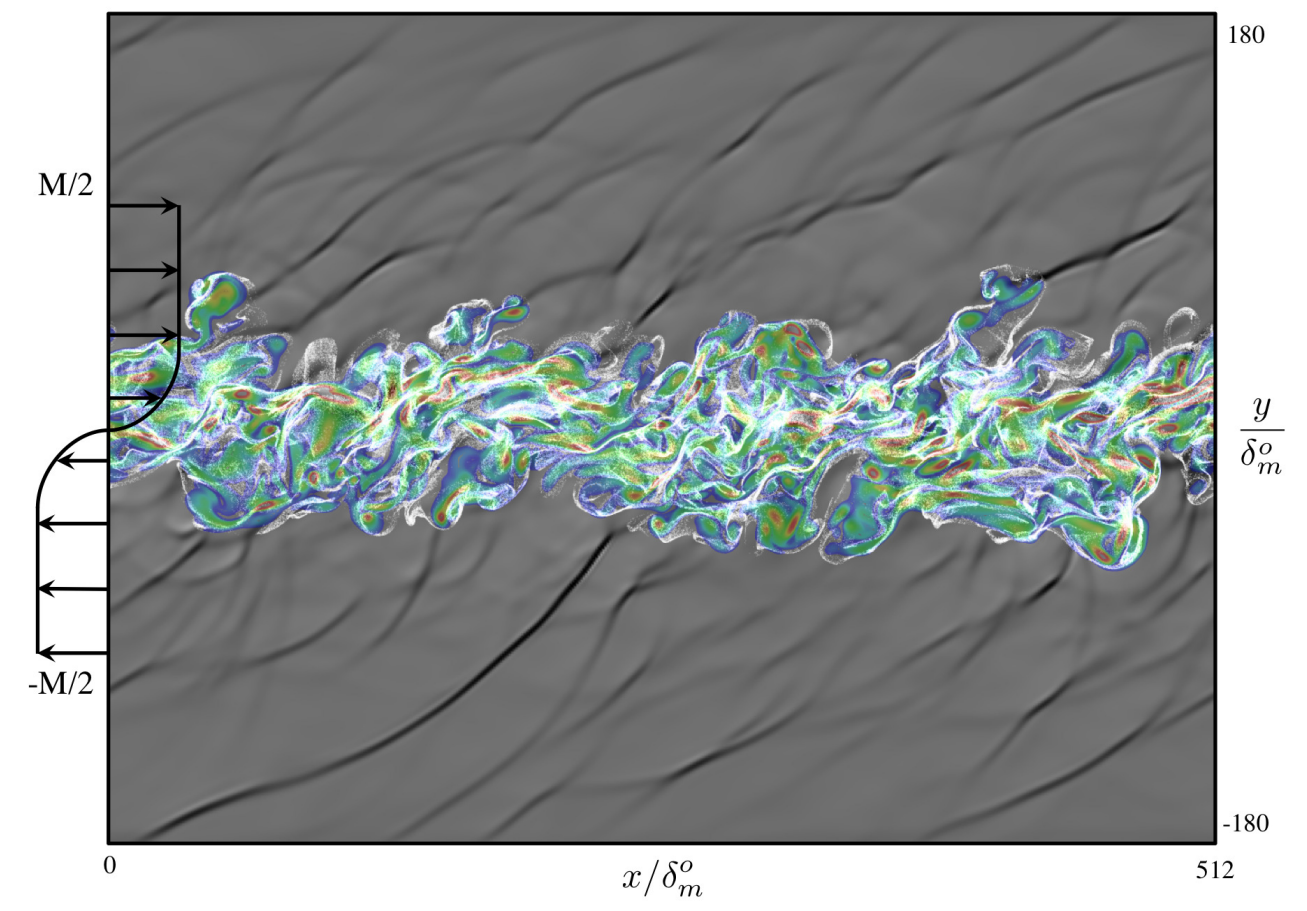

Figure 1. A higher speed, $M a=2.5$ temporally developing shear layer at $\delta_{m}^{o} / \Delta U=150$ showing the fluid velocity dilatation (grey scale), vorticity magnitude (color), and particle position (white).

Including the micro-jet impingement dynamics present in the near-nozzle region of the injector is challenging in the temporally developing shear layer considered herein. However, experimental measurements ${ }^{4}$ indicate that droplets break up quickly in the high-speed shear layer and reduce to a cloud of small (approximately monodisperse) droplets at the end of the injection region where the significant turbulence attenuation is observed. The present simulation configuration is designed to represent this post-injection region. Particles of equal size are initially seeded randomly throughout the shear layer very early in the simulation. The initial velocity of the particles is varied to assess the effect of mean-phase slip during the injection process. Owing to the moderately small Stokes numbers under consideration, the particles were found to respond to the flow quickly and attain an approximate self-similar state.

For each simulation presented herein, the domain length in the $x$ (streamwise), $y$ (vertical), and $z$ (spanwise) directions are taken to be $L_{x}=512 \delta_{m}^{o}, L_{y}=384 \delta_{m}^{o}$, and $L_{z}=128 \delta_{m}^{o}$ with $768 \times 771 \times 192$ grid points, respectively. These values were found to yield near-field pressure fluctuations of the single phase (unladen) case independent of the domain size. The Reynolds number based on the velocity difference between the upper and lower streams of the shear layer $\Delta U$ and the momentum thickness is taken to be $\operatorname{Re}_{\delta_{m}^{o}}=120$. The particle-to-fluid density ratio is taken to be that of water in air, i.e., $\rho_{p} / \rho_{f}=1000$. The particle diameter is chosen to match relevant Stokes numbers, defined as the ratio of particle response time 
to characteristic fluid time

$$
\mathrm{St} \equiv \tau_{p} / \tau_{f}=\frac{\rho_{p} d_{p}^{2} \operatorname{Re}_{\delta_{\omega}^{o}}}{18}
$$

where $\delta_{\omega}^{o}=4 \delta_{m}^{o}$ is the initial vorticity thickness of the shear layer. In this study we only consider St $=0.2$, corresponding to the value of the mean droplet diameter in the experiments of Krothapalli et al. ${ }^{4}$ The number of particles for each simulation case is determined based on the effective mass loading $M L$ within the shear layer, defined as

$$
M L=\frac{\rho_{p}}{\rho_{f}} \frac{N_{p} \pi d_{p}^{3} / 6}{L_{x} L_{z} \delta_{\omega}^{o}} .
$$

The mass loading is representative of the fraction of injected water to the total mass of the jet, and results in $N_{p}=25 \mathrm{M}$ for $M L=1$ to $N_{p}=50 \mathrm{M}$ for $M L=2$. Two initial conditions for the particle velocity are considered, particles at rest, i.e., $\boldsymbol{u}_{p}^{0}=0$, and an initial zero slip velocity between the phases, i.e., $\boldsymbol{u}_{p}^{0}=\boldsymbol{u}^{0}$, where $\boldsymbol{u}_{p}^{0}$ and $\boldsymbol{u}^{0}$ are the initial particle and fluid velocities, respectively. Simulation parameters for each case are provided in Table 1.

\begin{tabular}{lcccc}
\hline Case & $M L$ & St & $\boldsymbol{u}_{p}^{0}$ & $N_{p}$ \\
\hline Baseline & 0 (unladen) & - & - & 0 \\
Case 1 & 1 & 0.2 & 0 & $25 \times 10^{6}$ \\
Case 2 & 1 & 0.2 & $\boldsymbol{u}^{0}$ & $25 \times 10^{6}$ \\
Case 3 & 2 & 0.2 & 0 & $50 \times 10^{6}$ \\
\hline
\end{tabular}

Table 1. Simulation cases

\section{Simulation results}

\section{A. Shear layer growth and temporal evolution of $\left\langle p^{\prime} p^{\prime}\right\rangle$}

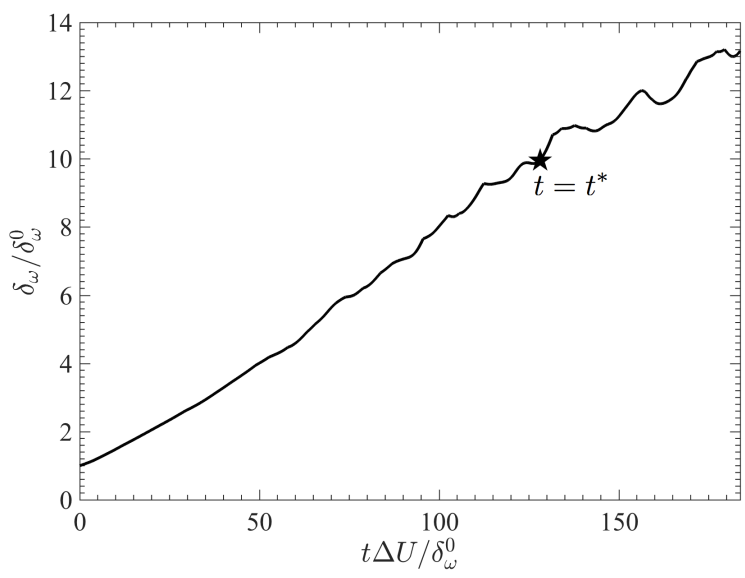

(a)

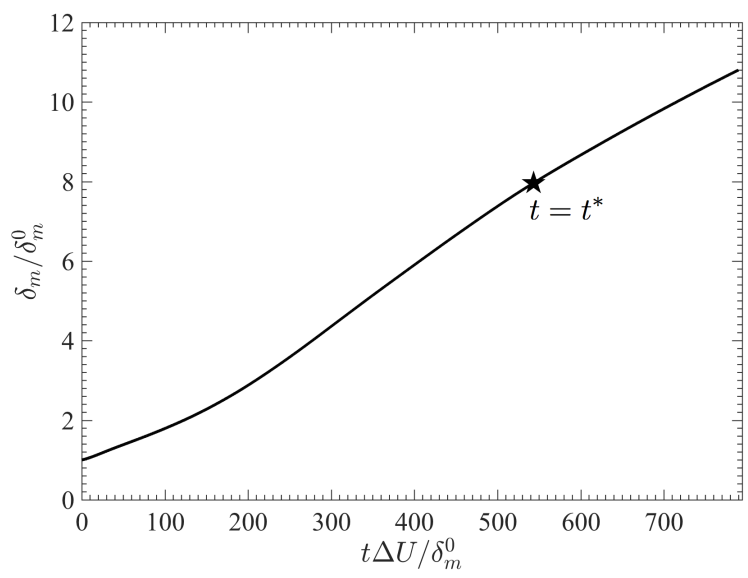

(b)

Figure 2. (a) Vorticity thickness as given by Eq. (14), and (b) momentum thickness Eq. (13) as a function of time for the Baseline (unladen) case. The star depicts the time at which particles are injected $\left(t^{*}\right)$.

After an initial transient, quasi-linear growth is observed in $\delta_{\omega}$ and $\delta_{m}$, as shown in Fig. 2. Here, the momentum thickness is defined as

$$
\delta_{m}=\frac{1}{\rho_{\infty} \Delta U^{2}} \int_{-\infty}^{\infty} \bar{\rho}\left(\frac{1}{4} \Delta U^{2}-\widetilde{u}^{2}\right) d y,
$$


where $\bar{\rho}$ is the mean density and $\tilde{u}$ the Favre-average streamwise velocity. The vorticity thickness, $\delta_{\omega}$, is given by

$$
\delta_{\omega}=\frac{\Delta U}{|d \tilde{u} / d y|_{\max }} .
$$

Once $\delta_{\omega}$ grew by a factor of ten, at time $t^{*}$ in Fig. 2, particles are randomly distributed within the vorticity thickness, i.e, $-\delta_{\omega}^{*} \leq y_{p} \leq \delta_{\omega}^{*}$. In the following sections, velocity and pressure statistics are reported, in addition to the overall sound reduction in the near field.

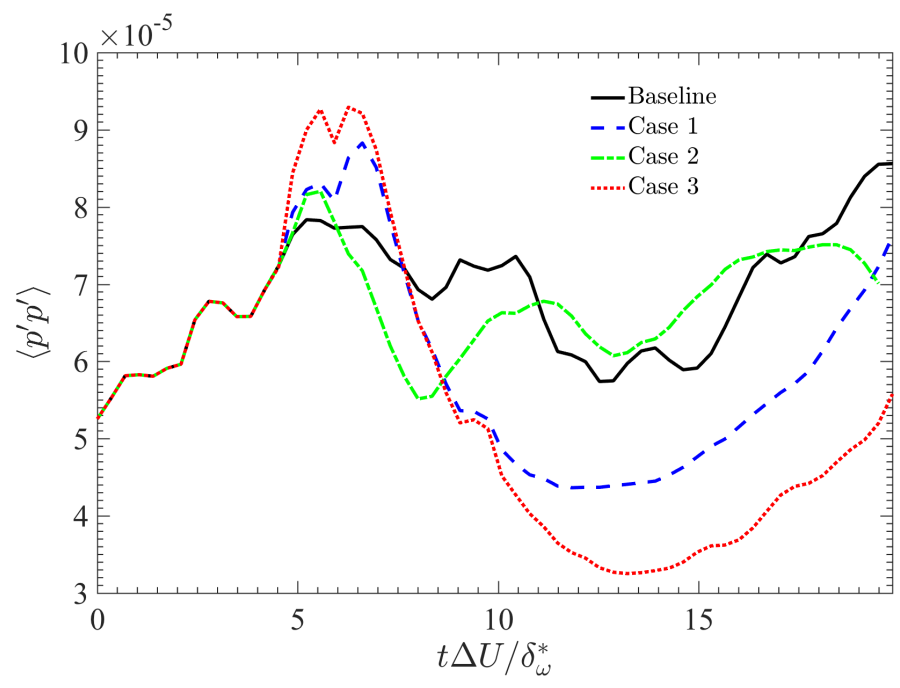

Figure 3. Temporal evolution of fluid-phase pressure intensity at $y=5.6 \delta_{\omega}^{*}$.

In this study, we are particularly interested in near-field noise generated by shear-induced turbulence, i.e., pressure fluctuations at a distance far from the mixing region of the shear layer. Figure 3 shows $\left\langle p^{\prime} p^{\prime}\right\rangle$ at a distance $y=5.6 \delta_{\omega}^{*}$ over the duration of the simulation. Here, angled brackets denote an average in the statistically homogeneous $\left(x\right.$ and $z$ ) directions, and fluctuating quantities are defined as $(\cdot)^{\prime}=(\cdot)-\langle(\cdot)\rangle$. The value of $y=5.6 \delta_{\omega}^{*}$ was chosen to represent the furthest distance from the shear layer while avoiding effects near the boundary. After seeding the mixing layer with particles, it can be seen that it takes approximately $5 \Delta U / \delta_{\omega}^{*}$ for information to propagate to this location. Case 3 (the shear layer laden with the highest mass loading) has the largest effect on the pressure fluctuations, with a reduction of about half that of the unladen case at $t \Delta U / \delta_{\omega}^{*} \approx 13$. Case 1 ( $M L=1$ with initial zero particle velocity) also exhibits a strong reduction in pressure fluctuations a this time. It is interesting to note that Case $2(M L=1$ with initial zero slip velocity) does not appear to modify $\left\langle p^{\prime} p^{\prime}\right\rangle$. It can therefore be concluded that the initial velocity of the particles (i.e., the model for particle injection velocity) has an order-one effect on this quantity, suggesting perhaps a sensitivity to the injection-dynamics in the target configuration.

\section{B. Visualization}

An instantaneous view of the local particle concentration is shown in Fig. 4. Distinct clustering can be observed with local concentrations exceeding 40 times the mean value. The clusters are seen to exhibit preferential alignment with the flow. Visualizations of velocity dilatation at $t \Delta U / \delta_{\omega}^{*}=20$ of the unladen and $M L=1$ case with $\boldsymbol{u}_{p}^{0}=0$ are shown in Fig. 5, corresponding to the unladen flow and Case 1 in Table 1. The particle loading in contrast to the unladen case appears to reduce the finer scale fluctuations in the turbulence and the near-field above the turbulence. The corresponding reduction in these pressure fluctuations are quantified in the following section.

\section{Particle-laden effects on the turbulence}

We now examine the extent to which particles affect the fluid-phase velocity fluctuations. Root-mean-square (RMS) velocity fluctuations are shown in Fig. 6. The presence of particles reduce the velocity fluctuations 


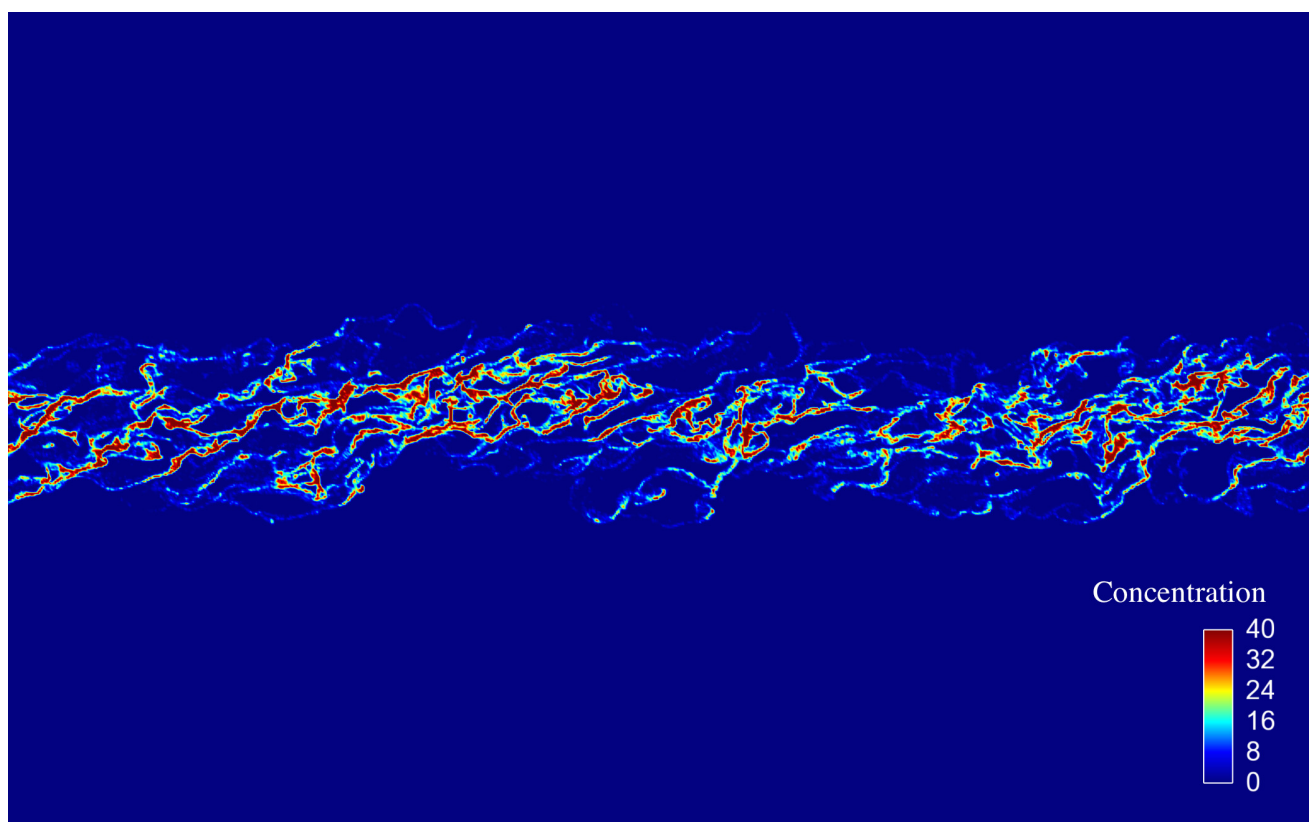

Figure 4. Zoomed in view of a two-dimensional plane of the shear layer showing instantaneous particle concentration normalized by the mean concentration.

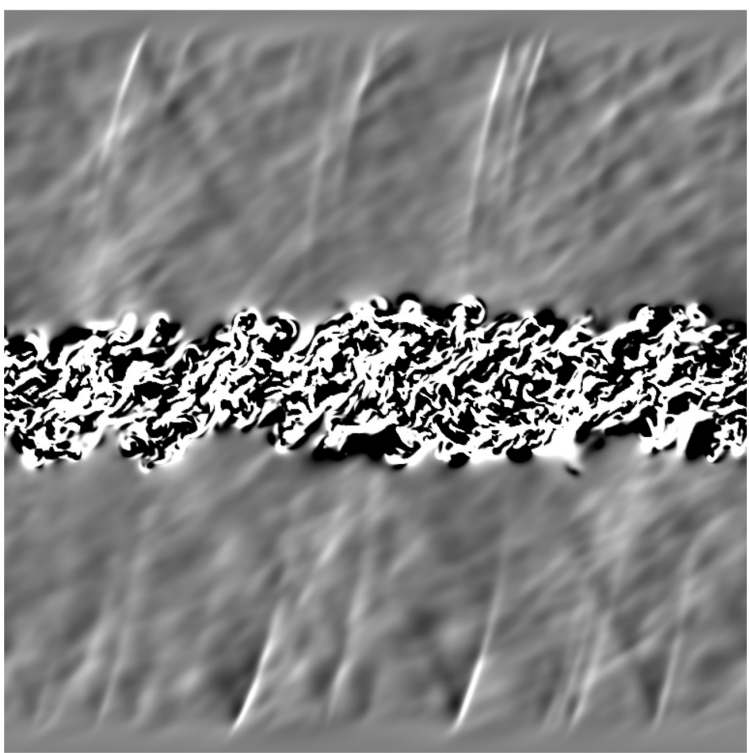

(a)

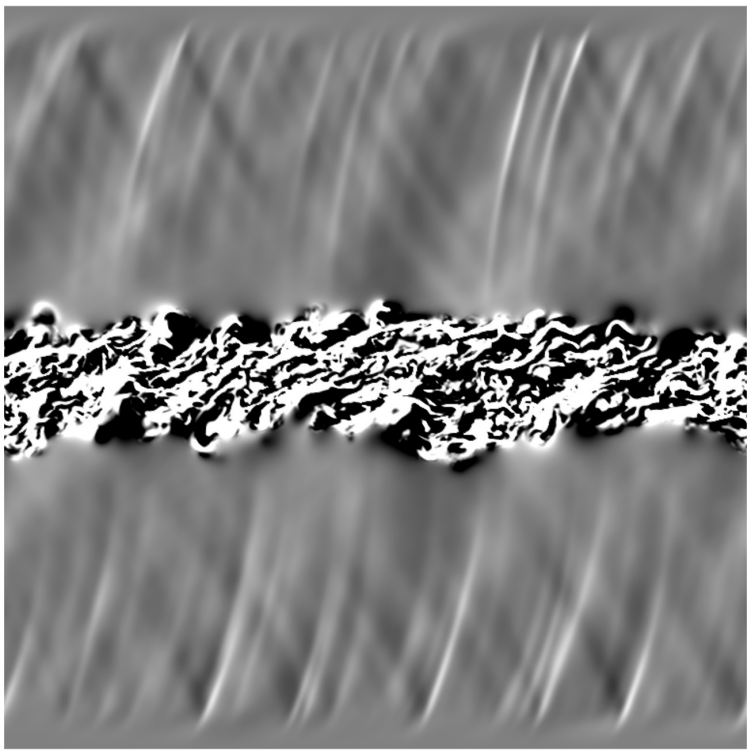

(b)

Figure 5. Visualization of fluid velocity dilatation $(\nabla \cdot \boldsymbol{u})$ of an $x-y$ plane at $t \Delta U / \delta_{\omega}^{*}=20$ of the (a) unladen case and (b) $M L=1$ with $u_{p}^{0}=0$. 
within the shear layer $\left(y<\delta_{\omega}\right)$ with the greatest effect at the midplane $(y=0)$. As with the pressure fluctuation profiles, the greatest reduction in fluid-phase turbulent kinetic energy (TKE) is attributed to Case 3, with Case 1 also resulting in a large modification compared to the unladen case. Although Case 2 was observed to have a small effect on $\left\langle p^{\prime} p^{\prime}\right\rangle$, even with zero initial slip the particles-turbulence coupling reduces the overall TKE in the fluid by $\approx 42 \%$ at the centerline. At $y<\delta_{\omega} / 2$, all cases are seen to reduce the velocity fluctuations in each direction. Interestingly, at $\delta_{\omega} / 2<y<\delta_{\omega}$, Cases 2 and 3 are seen to increase the velocity fluctuations above the unladen case.

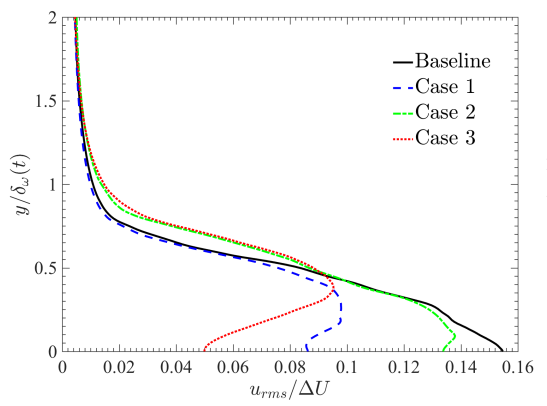

(a)

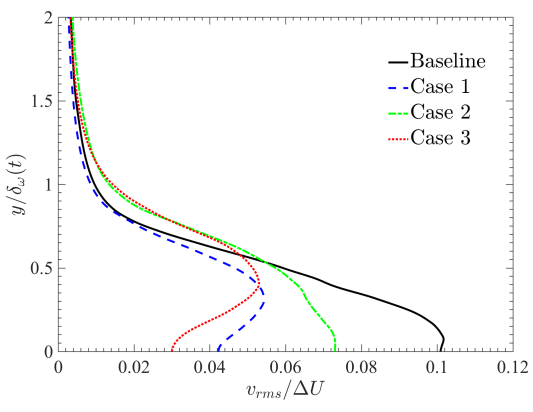

(b)

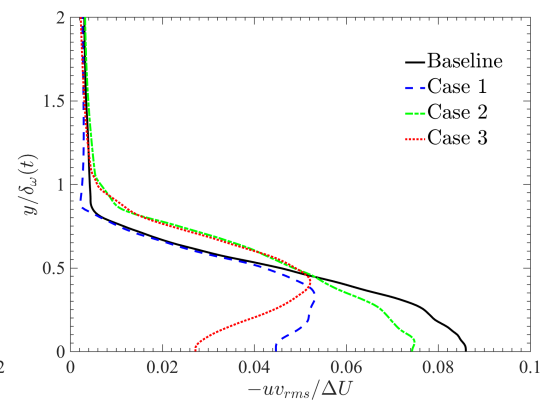

(c)

Figure 6. Variation of the root-mean-square velocity fluctuations (a) $u_{r m s}$, (b) $v_{r m s}$, and (c) $-u v_{r m s}$ with distance from the mixing layer.

\section{Particle-laden effects on the near-field pressure fluctuations}

Figure 7(a) shows the mean pressure intensity as a function of $y$. Greatest reduction in pressure fluctuations are seen to occur within the shear layer $\left(y \lesssim 1 \delta_{w}\right)$ where particle-turbulence interactions occur. However, of key interest in this work is to what extent particle interactions in the shear layer affect noise radiated in the near field. Figure 7(b) shows the change in sound pressure level compared to the unladen case above the shear layer $\left(y>\delta_{\omega}\right)$. Case 1 is seen to have a maximum reduction of approximately $2 \mathrm{~dB}$, and Case 4 results in greater than $2.5 \mathrm{~dB}$ reduction. While a small reduction is observed in Case 2, the overall change is far smaller than the cases with finite initial slip velocity. This suggests that for small Stokes numbers, the majority of noise reduction may depend on the initial injection event before the droplets relax to the local flow. Ongoing simulations and future results will report on the Stokes number and Mach number effect on the radiated noise.

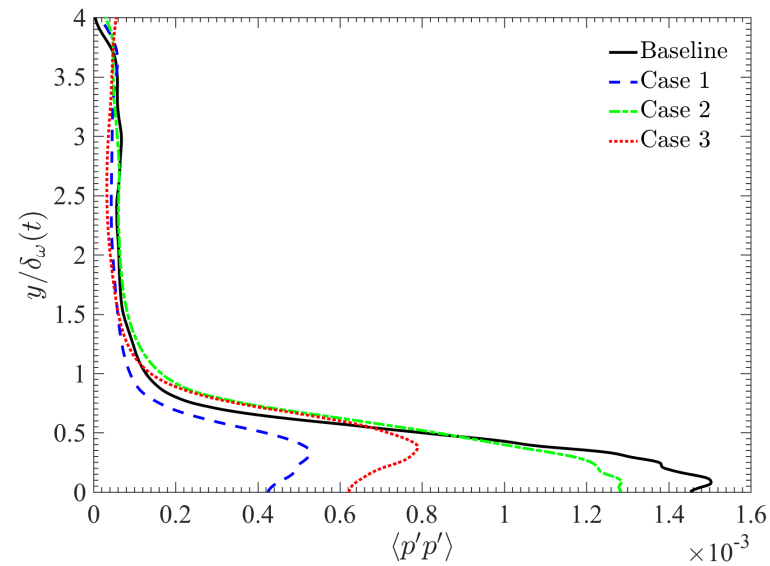

(a)

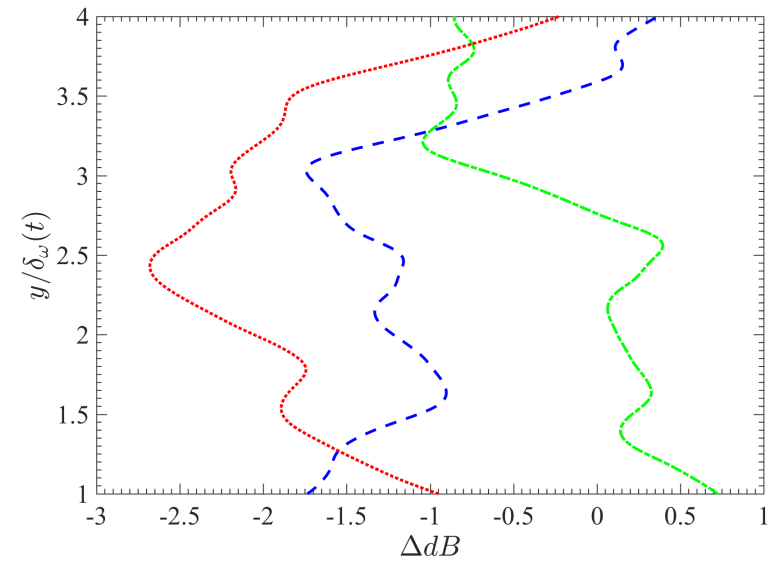

(b)

Figure 7. (a) Mean pressure intensity and (b) change in sound level compared to the Baseline as a function of distance from the mixing layer. 


\section{Conclusions}

Direct numerical simulations of $M a=1.5$ free-shear-flow turbulence laden with finite size inertial droplets were performed to study the effect of particle-turbulence interactions on radiated sound. Results suggest that while reduction in near-field pressure fluctuations is linked to mass loading, the particle injection velocity has a greater overall effect. A maximum sound reduction in the near field of $2.53 \mathrm{~dB}$ was observed for the highest mass loading case. It remains to be seen, however, to what extent particles affect radiated sound at different Mach numbers and Stokes numbers. In addition, accounting for thermal effects (e.g., evaporation and heat transfer) will modify the local sound speed and is anticipated to have a significant effect on radiated pressure fluctuations. While further quantitative comparisons with available experimental data is necessary to properly validate the various modeling components, the present study demonstrates the capability of numerical simulations to capture particle-turbulence-acoustic coupling leading to near-field sound reduction.

\section{Acknowledgment}

A portion of the baseline simulation framework was developed by D. A. Buchta while a student of Professor Jonathan Freund at the University of Illinois. The fluid-phase solver used in this work was based on the compressible flow solver developed by Ramanathan Vishnampet also under the advisement of Prof. Freund. This research used resources of the Oak Ridge Leadership Computing Facility, which is a DOE Office of Science User Facility supported under Contract DE-AC05-00OR22725.

\section{References}

${ }^{1}$ Zoppellari, E. and Juve, D., "Reduction of jet noise by water injection," AIAA/CEAS Aeroacoustics Conference, Atlanta, GA, 1997, pp. 266-274.

${ }^{2}$ Ikawa, H. and Laspesa, F., "Analytical understanding of WTR ignition/duct overpressure induced by space shuttle solid rocket motor ignition transient," 19th Joint Propulsion Conference, 1983, p. 1113.

${ }^{3}$ Zoppellari, E. and Juve, D., "Reduction of hot supersonic jet noise by water injection," AIAA paper, Vol. 2204, 1998, pp. 1998.

${ }^{4}$ Krothapalli, A., Venkatakrishnan, L., Lourenco, L., Greska, B., and Elavarasan, R., "Turbulence and noise suppression of a high-speed jet by water injection," Journal of Fluid Mechanics, , No. 491, 2003, pp. 131-159.

${ }^{5}$ Norum, T. D., "Reductions in multi-component jet noise by water injection," AIAA paper, Vol. 2976, 2004 , pp. 2004.

${ }^{6}$ Greska, B. and Krothapalli, A., "Jet noise reduction using aqueous microjet injection," AIAA paper, Vol. $2971,2004$.

${ }^{7}$ Krothapalli, A., Greska, B., and Arakeri, V., High-Speed Jet Noise Reduction Using Microjets, 2005.

${ }^{8}$ Lighthill, M. J., "On sound generated aerodynamically. I. General theory," Proceedings of the Royal Society of London A: Mathematical, Physical and Engineering Sciences, Vol. 211, No. 1107, 1952, pp. 564-587.

${ }^{9}$ Ffowcs Williams, J. E., "The noise from turbulence convected at high speed," Philosophical Transactions of the Royal Society of London, Vol. 255, 1963, pp. 469-503.

${ }^{10}$ Ffowcs Williams, J. E., Simson, J., and Virchis, V. J., "“Crackle': An annoying component of jet noise." Journal of Fluid Mechanics, Vol. 71, 1975, pp. 251-271.

${ }^{11}$ Buchta, D. A., Anderson, A. T., and Freund, J. B., "Near-field shocks radiated by high-speed free-shear-flow turbulence," 20th AIAA/CEAS Aeroacoustics Conference, 2014.

${ }^{12}$ Squires, K. D. and Eaton, J. K., "Preferential concentration of particles by turbulence," Phys. Fluids, Vol. 3, 1991, pp. 1169 .

${ }^{13}$ Eaton, J. K. and Fessler, J. R., "Preferential concentration of particles by turbulence," Int. J. Multiphase Flow, Vol. 20, 1994, pp. 169-209.

${ }^{14}$ Reade, W. C. and Collins, L. R., "Effect of preferential concentration on turbulent collision rates," Phys. Fluids, Vol. 12, 2000, pp. 2530.

${ }^{15}$ Aliseda, A., Cartellier, A., Hainaux, F., and Lasheras, J. C., "Effect of preferential concentration on the settling velocity of heavy particles in homogeneous isotropic turbulence," J. Fluid Mech., Vol. 468, 2002, pp. 77-105.

${ }^{16}$ Ray, B. and Collins, L. R., "Preferential concentration and relative velocity statistics of inertial particles in Navier-Stokes turbulence with and without filtering," J. Fluid Mech., Vol. 680, 2011, pp. 488-510.

${ }^{17}$ Ireland, P. J. and Collins, L. R., "Direct numerical simulation of inertial particle entrainment in a shearless mixing layer," Journal of Fluid Mechanics, Vol. 704, 2012, pp. 301-332.

${ }^{18}$ Sundaram, S. and Collins, L. R., "A numerical study of the modulation of isotropic turbulence by suspended particles," J. Fluid Mech., Vol. 379, 1999, pp. 105-143.

${ }^{19}$ Balachandar, S. and Eaton, J. K., "Turbulent dispersed multiphase flow," Annu. Rev. Fluid Mech., Vol. 42, 2010, pp. 111-133.

${ }^{20}$ Capecelatro, J., Desjardins, O., and Fox, R. O., "Numerical study of collisional particle dynamics in cluster-induced turbulence," J. Fluid Mech., Vol. 747, 2014, pp. R2 1-13.

${ }^{21}$ Capecelatro, J., Desjardins, O., and Fox, R. O., "On fluid-particle dynamics in fully developed cluster-induced turbulence," Journal of Fluid Mechanics, Vol. 780, 2015, pp. 578-635. 
${ }^{22}$ Xia, Z., Shi, Y., Zhang, Q., and Chen, S., "Modulation to compressible homogenous turbulence by heavy point particles. I. Effect of particles' density," Physics of Fluids (1994-present), Vol. 28, No. 1, 2016, pp. 016103.

${ }^{23}$ Clift, R., Grace, J., and Weber, M., Bubbles, drops, and particles, Academic Press Incorporated, 1978.

${ }^{24}$ Yeung, P. and Pope, S., "An algorithm for tracking fluid particles in numerical simulations of homogeneous turbulence," Journal of computational physics, Vol. 79, No. 2, 1988, pp. 373-416.

${ }^{25}$ Horwitz, J. and Mani, A., "Accurate calculation of Stokes drag for point-particle tracking in two-way coupled flows," J. Comp. Phys., Vol. 318, 2016, pp. 85-109.

${ }^{26}$ Sundaram, S. and Collins, L. R., "Numerical considerations in simulating a turbulent suspension of finite-volume particles," J. Comp. Phys., Vol. 142, 1996, pp. 337-350.

${ }^{27}$ Strand, B., "Summation by parts for finite difference approximations for d/dx," Journal of Computational Physics, Vol. 110, No. 1, 1994, pp. 47-67.

${ }^{28}$ Bodony, D. J., "Accuracy of the simultaneous-approximation-term boundary condition for time-dependent problems," Journal of Scientific Computing, Vol. 43, No. 1, 2010, pp. 118-133.

${ }^{29}$ Mattsson, K., Svärd, M., and Nordström, J., "Stable and accurate artificial dissipation," Journal of Scientific Computing, Vol. 21, No. 1, 2004, pp. 57-79.

${ }^{30}$ Vishnampet Ganapathi Subramanian, R., An exact and consistent adjoint method for high-fidelity discretization of the compressible flow equations, Ph.D. thesis, University of Illinois at Urbana-Champaign, 2015.

${ }^{31}$ Buchta, D. A., Crackle noise from high-speed free-shear-flow turbulence, Ph.D. thesis, University of Illinois at UrbanaChampaign, 2016.

${ }^{32}$ Kleinman, R. and Freund, J. B., "The sound from mixing layers simulated with different ranges of turbulence scales," Physics of Fluids, Vol. 20, No. 10, 2008.

${ }^{33}$ Lui, C., A numerical investigation of shock-associated noise, Ph.D. thesis, Stanford University, 2003.

${ }^{34}$ Kim, J., Bodony, D. J., and Freund, J. B., "Adjoint-based control of loud events in a turbulent jet," Journal of Fluid Mechanics, Vol. 741, 2 2014, pp. 28-59.

\section{Appendix}

\section{A. Initial condition for the fluid phase}

Perturbations are introduced to the fluid-phase variables to ensure the flow develops into realistic, broadband turbulence with an approximately self-similar state before seeding the shear layer with the water-density model particles. We begin with a Fourier-based scalar field, similar to the initial excitation in previous mixing-layer simulations, ${ }^{32}$ as

$$
a(\boldsymbol{x}, t)=\sum_{i=1}^{n_{k_{x}}} \sum_{k=-n_{k_{z}}}^{n_{k_{z}}} \cos \left(k_{x}^{i} x+k_{y}^{o} y+k_{z}^{k} z+\theta_{i, k}\right)
$$

with streamwise, normal, and spanwise wavenumbers

$$
k_{x}^{i}=\frac{\pi}{L_{x}} i, k_{y}^{o}=\frac{2 \pi}{10 \delta_{m}^{o}}, k_{z}^{k}=\frac{2 \pi}{L_{z}} k,
$$

respectively. The number of modes in the streamwise and spanwise direction are set to $n_{k_{x}}=10$ and $n_{k_{z}}=5$. Each corresponding phase, $\theta_{i, k}$, in (15) is set to a psuedo-random number between $[-\pi, \pi]$. To disrupt the harmonic character of the scalar field in (15), we introduce conditions that randomly 'jitter' the initial phases in a manner similar to previous compressible turbulence simulations. ${ }^{33,34}$ We assign discrete regions in the computational domain of size $\left(L_{x} / 5 \times L_{y} \times L_{z} / 5\right)$ a random number $r$ between [0,1]. The phases in (15) are then modified by the following rules

$$
\phi_{i, k}= \begin{cases}\phi_{i, k}+\pi & r<\frac{1}{3} \\ \phi_{i, k}-\pi & \frac{1}{3} \leq r<\frac{2}{3} \\ \phi_{i, k} & \frac{2}{3} \leq r<1 .\end{cases}
$$

The solenoidal fluctuating velocity field is set by numerically solving the cross-product,

$$
\boldsymbol{v}^{\prime}=\nabla \times[a, a, a],
$$

which is then mollified to be supported within the shear layer thickness $y= \pm \delta_{w}^{o}$ by

$$
\boldsymbol{w}^{\prime}=\boldsymbol{v}^{\prime} \times \frac{1}{2}\left[\tanh \left(\frac{5}{\delta_{w}^{o}}\left(y+\delta_{w}^{o}\right)\right)-\tanh \left(\frac{5}{\delta_{w}^{o}}\left(y-\delta_{w}^{o}\right)\right)\right] .
$$


The velocity fluctuations in (19) are then normalized by their average root-mean-square level across the shear layer and re-scaled to $7.5 \%$ of the $\Delta U$ velocity difference by

$$
\boldsymbol{u}^{\prime}=0.075 \cdot \Delta U \cdot \frac{\boldsymbol{w}^{\prime}}{u_{r m s}}
$$

with

$$
u_{r m s}=\left\langle w_{1}^{\prime} w_{1}^{\prime}+w_{2}^{\prime} w_{2}^{\prime}+w_{3}^{\prime} w_{3}^{\prime}\right\rangle^{0.5} .
$$

The $\langle\cdot\rangle$ operator in (21) is an average of the fluctuations over $\left(-\delta_{m}^{o} \leq y \leq \delta_{m}^{o}\right)$ and over the streamwise- and spanwise-periodic directions. The velocity fluctuations in (20) are added to the mean velocity as

$$
\boldsymbol{u}=\boldsymbol{u}^{\prime}+[\bar{u}(y), 0,0],
$$

where

$$
\bar{u}(y)=\frac{\Delta U}{2} \tanh \left(\frac{y}{2 \delta_{w}^{o}}\right) .
$$

The mean density is set by the Crocco-Busemann relation

$$
\bar{\rho}(y)=\rho_{\infty}\left[\frac{(\gamma-1)}{2 c_{\infty}^{2}}\left(\frac{\Delta U^{2}}{4}-\bar{u}(y)^{2}\right)+1\right]^{-1},
$$

and the mean pressure is set to a constant $\bar{p}=1 / \gamma$. 